Original paper

\title{
Preparing data for multiparametric PET/MR imaging: Influence of PET point spread function modelling and EPI distortion correction on the spatial correlation of $\left[{ }^{18} \mathrm{~F}\right]$ FDG-PET and diffusion-weighted MRI in head and neck cancer
}

\author{
Anders Olin $^{\mathrm{a}, *, 1}$, Louise Krogager ${ }^{\mathrm{a}, 1}$, Jacob H. Rasmussen ${ }^{\mathrm{b}}$, Flemming L. Andersen ${ }^{\mathrm{a}}$, Lena Specht ${ }^{\mathrm{c}}$, \\ Thomas Beyer $^{\mathrm{a}, \mathrm{e}}$, Andreas Kjaer ${ }^{\mathrm{a}, \mathrm{d}}$, Barbara M Fischer ${ }^{\mathrm{a}, \mathrm{f}}$, Adam E. Hansen ${ }^{\mathrm{a}}$ \\ ${ }^{a}$ Department of Clinical Physiology, Nuclear Medicine and PET, Rigshospitalet, University of Copenhagen, Copenhagen, Denmark \\ ${ }^{\mathrm{b}}$ Department of Otorhinolaryngology, Head \& Neck Surgery and Audiology, Rigshospitalet, University of Copenhagen, Copenhagen, Denmark \\ ${ }^{\mathrm{c}}$ Department of Oncology, Section Radiotherapy, Rigshospitalet, University of Copenhagen, Copenhagen, Denmark \\ ${ }^{\mathrm{d}}$ Cluster for Molecular Imaging, University of Copenhagen, Copenhagen, Denmark \\ e QIMP Team, CMPBME, Medical University of Vienna, Austria \\ ${ }^{\mathrm{f}}$ PET Centre, School of Biomedical Engineering and Imaging Sciences, Kings College London, UK
}

A R T I C L E I N F O

\section{Keywords:}

Multiparametric PET/MR

DWI distortion correction

PSF modelling

\begin{abstract}
A B S T R A C T
Purpose: Multiparametric imaging holds great potential for characterization of disease heterogeneity. For integrated PET/MR imaging, the combination of ${ }^{18} \mathrm{~F}$-flourodeoxyglucose (FDG) PET and diffusion weighted imaging (DWI) has been suggested for the assessment of tumor heterogeneity. However, PET image resolution is limited and DWI is prone to image distortions. The aim of this study was to assess the influence of PET point spread function (PSF) modelling and DWI distortion correction on the voxelwise correlation between FDG-PET and DWI.

Methods: Data were collected from 11 patients with head and neck cancer, each undergoing PET/MR imaging twice. PET reconstructions with and without PSF modelling and DWI with and without distortion correction were derived. Tumor SUV was compared between PET reconstructions by linear regression. Geometric distortions of DWI with and without distortion correction were quantified by voxelwise correlation coefficients to an undistorted anatomical reference. The influence of PSF modelling and DWI distortion correction on a multiparametric analysis was assessed as a change of the voxelwise correlation coefficient between FDG-PET and DWI measured in tumors.

Results: The inclusion of PSF modelling in the PET reconstruction affected tumor quantification by a 10-20\% increase in SUV. Distortion correction reduced DWI geometric distortions significantly. The impact of PET PSF modelling on the spatial correlation with DWI was insignificant. However, distortion correction of DWI had a significant effect on the spatial correlation with PET.

Conclusions: Proper preparation of the imaging modalities is important for a correct analysis and interpretation of multiparametric PET/MR imaging of head and neck cancer.
\end{abstract}

\section{Introduction}

In the area of personalized cancer medicine, assessment of tumor heterogeneity plays an important role as it allows for the selection of more effective and targeted treatments that, in turn, may extend survival of patients [1]. Imaging offers a non-invasive assessment of tumor heterogeneity. In this respect, multiparametric imaging has become an area of great interest, since the combination of modalities may yield a more precise characterization of tumor properties. An example of such a combination is ${ }^{18} \mathrm{~F}$-flourodeoxyglucose (FDG) PET and diffusionweighted imaging (DWI). While both modalities are sensitive for the detection of cancer, they utilize different biological properties of the

${ }^{*}$ Corresponding author at: Department of Clinical Physiology, Nuclear Medicine and PET, Rigshospitalet, University of Copenhagen, Blegdamsvej 9 , 2100 Copenhagen, Denmark.

E-mail address: anders.olin@regionh.dk (A. Olin).

${ }^{1}$ These authors contributed equally. 
malignant tissue. FDG-PET is an indicator of glucose metabolism, which is often increased in malignant tumors, while DWI assesses the movement of water molecules and characterizes cellular density [2]. It is suggested that FDG-PET - by means of standardized uptake value (SUV) - and DWI - expressed as apparent diffusion coefficient (ADC) - correlate weakly, but with a statistically significant negative correlation on a voxel level within tumors of individual patients $[3,4]$. The voxelwise combination of SUV and ADC may further be used to identify tissue classes within the tumor by joint statistical modelling [5-9]. With the introduction of integrated PET/MR, it has become possible to acquire PET and DWI simultaneously, thereby potentially improving image coregistration. However, several physical factors restrict the spatial resolution of PET images, while DWI can be geometrically distorted due to magnetic field inhomogeneities, which may compromise the spatial correspondence of PET and DWI. This could influence a combined voxelwise analysis of the SUV and ADC.

The limited spatial resolution of PET images can be addressed using point-spread function (PSF) modelling. Here, the response of the PET system is modelled [10] and included into the image reconstruction process [11,12]. PSF modelling is widely used in PET/CT and has proven useful for oncologic purposes [13]. PSF modelling is now also available for integrated PET/MR, but so far has been evaluated in only two studies [14,15].

DWI is usually performed by the technique of echo-planar imaging (EPI), which is prone to distortions [16]. Distortions may occur on boundaries between regions of different magnetic susceptibility and hence, the degree of distortions depends on anatomic region [17]. For head and neck cancer, the complex anatomy enhances magnetic field inhomogeneities and, thus, EPI distortions [18]. Optimization of DWI acquisition can reduce distortions, employing e.g. parallel imaging, zoomed field-of-view [18] and read-out segmentation [19]. Distortions may also be corrected with post-processing techniques, e.g., based on $\mathrm{B}_{0}$-field mapping [20]. Such techniques have frequently been utilized for brain fMRI [21] but only very little in context of oncology $[8,22]$.

Therefore, both PSF modelling for PET image reconstruction and distortion correction of DWI can potentially improve PET/MR image quality and, thus, be expected to provide a robust basis for a voxelwise combination of the modalities.

This study evaluates the effect of PSF modelling and EPI distortion correction in the setting of multiparametric imaging of head and neck cancer performed with simultaneous PET/MR. More specifically, we investigate the influence of PSF modelling and EPI distortion correction on the spatial correlation between FDG-PET and DWI.

\section{Materials and methods}

\subsection{Patients}

Eleven patients with head and neck cancer were included in this study. Each patient was scanned twice exactly 3 days apart. Patients were recruited as part of a reproducibility study [23] and feasibility study [8], where patients underwent PET/CT prior to PET/MR. The 11 patients included in the present study were based on the availability of an optimized DWI sequence (see below). The study was approved by the local ethics committee (approval no. H-3-2012-072).

\subsection{Image acquisition}

All patients were scanned on an integrated PET/MR system (Siemens Biograph mMR) using a 16-channel receive head and neck MR coil. Patients were instructed to fast for minimum $6 \mathrm{~h}$ before the exam and were scanned 100-120 min after injection of FDG ( $4 \mathrm{MBq} / \mathrm{kg}$ ). PET was performed as a single-bed, 20 min acquisition. PET images were reconstructed using OP-OSEM with 3 iterations, 21 subsets and $4 \mathrm{~mm}$ Gaussian post-filter. PET images was also reconstructed using PSF modelling (PSF OP-OSEM) with 3 iterations, 21 subsets and $2 \mathrm{~mm}$
Gaussian post-filter. The vendor recommends a decrease in Gaussian post filter size from $4 \mathrm{~mm}$ to $2 \mathrm{~mm}$ when using PSF modelling [15]. All images were reconstructed on $344 \times 344$ matrices with a pixel size of $2 \times 2 \mathrm{~mm}^{2}$ and slice thickness of $2 \mathrm{~mm}$.

A standard DWI was acquired with a 2-dimensional EPI sequence using a matrix size of $128 \times 128$ with varying number of slices depending on patient and a pixel size of $1.95 \times 1.95 \mathrm{~mm}^{2}$ with a slice thickness/gap of $4.0 / 0.4 \mathrm{~mm}$, flip angle of $90^{\circ}$, repetition time/echo time of $5400 / 84 \mathrm{~ms}$, a parallel imaging factor of 2 , an effective echo spacing of $0.375 \mathrm{~ms}$ and performed with 3 different $b$-values $(0,500$, $1000 \mathrm{~s} / \mathrm{mm}^{2}$ ).

To reduce distortions, an optimized DWI protocol was developed by changing the following settings: repetition time of $3000 \mathrm{~ms}$, parallel imaging factor of 4 and an effective echo spacing of $0.145 \mathrm{~ms}$. It was measured as 3 stacks with 8 slices each, each stack at the isocenter of the scanner to maximize field homogeneity. The matrix size of the optimized DWI was $92 \times 92 \times 24$, with a pixel size of $2.71 \times 2.71 \mathrm{~mm}^{2}$ and a slice thickness/gap of 4.0/0.4 mm. For $\mathrm{B}_{0}$-field mapping, double echo sequences with a repetition time of $100 \mathrm{~ms}$ and echo times of 4.82 and $7.38 \mathrm{~ms}$ were acquired in 3 stacks and with matrix size and voxel size as the optimized DWI. Serving as a reference for the DWI, an anatomical T2-weighted MR sequence with fat suppression by short tau inversion recovery (STIR) was acquired. All DWI sequences were performed during the PET acquisition. Additional MR images were obtained in the scan protocol but is not used here [23].

\subsection{Image processing}

EPI distortions were corrected using the algorithm FMRIB's Utility for Geometrically Unwarping EPIs (FUGUE) of the FSL software package (Analysis Group, FMRIB where FMRIB is Oxford Centre for Functional MRI of the Brain) [22]. FUGUE reduces artefacts by using a computed $\mathrm{B}_{0}$-field map based on two collected gradient echo images and a corresponding phase map. The distortion corrected DWIs were fitted to a mono-exponential function with regard to $b$-values using DTIFit, from the FSL toolkit. All PET reconstructions, standard DWI and MR-STIR were resampled to the dimensions of the optimized DWI in order to do voxelwise analysis. Resampling was carried out using Medical Imaging NetCDF (MINC) (Montreal Neurological Institute).

\subsection{VOI definition}

A volume of interest (VOI) of each tumor was delineated on the PET OP-OSEM $4 \mathrm{~mm}$ image by a PET/MR-experienced physician using the image analysis tool Mirada XD (MiradaMedical). The VOIs were based on a $40 \%$ of $\mathrm{SUV}_{\max }$ isocontour. Thresholding adjustments aiming at obtaining the steepest gradient between tumor and background, as well as manual alterations necessary to exclude adjacent physiologic uptake were performed if considered appropriate by the physician. Subsequently, for each tumor, a $4 \mathrm{~cm}$-diameter sphere VOI with the center located at the center of mass of the tumor was created.

\subsection{FDG-PET analysis}

For each lesion, $\mathrm{SUV}_{\text {mean }}$ and $\mathrm{SUV}_{\text {max }}$ were extracted from the entire tumor VOIs and compared between the two PET reconstructions (OPOSEM $4 \mathrm{~mm}$ and PSF OP-OSEM $2 \mathrm{~mm}$ ) using simple linear regression with an intercept fixed at zero. The relative difference in $\mathrm{SUV}_{\text {mean }}$ between the two reconstruction types as a function of tumor size was assessed using simple linear regression.

\subsection{DWI analysis}

Three types of DWI were available: Standard, optimized and optimized with EPI distortion correction by FUGUE. The distortions were quantitatively evaluated by the voxelwise correlation coefficients 
between the DWI with $b$-value $=0 \mathrm{~s} / \mathrm{mm}^{2}$ and the anatomical reference (STIR) within the $4 \mathrm{~cm}$-diameter sphere. These two images have comparable contrast, as both sets of images are T2-weighted with fat suppression.

\subsection{Joint FDG-PET and DWI analysis}

The spatial correlation of FDG-PET and DWI were studied by calculating the voxelwise correlation coefficients between SUV and ADC within the tumor VOIs in individual patients. First, the influence of PSF modelling was studied: The voxelwise correlation coefficients between SUV and ADC were compared for PET reconstructions with OP-OSEM $4 \mathrm{~mm}$ and PSF OP-OSEM $2 \mathrm{~mm}$. DWI was in both cases optimized and distortion corrected with FUGUE (best expected quality). Second, the influence of distortion correction was studied: The voxelwise correlation coefficients between SUV and ADC were compared between optimized DWI and optimized DWI including with distortion correction by FUGUE. PET was in both cases reconstructed with PSF modelling (best expected quality).

Finally, Bland-Altman analyses of the reproducibility of the spatial correlation of FDG-PET and DWI between the two patient exams were carried out. The limits of agreement and 95\% confidence intervals (CI) of the limits of agreement estimates were calculated [24]. The reproducibility of the voxelwise correlation coefficient between SUV and ADC was studied for all combinations of PET reconstructed with and without PSF modelling and DWI with and without distortion correction.

\subsection{Statistics}

Voxelwise correlations were computed as Spearman's rank correlation coefficients. Differences of the correlation coefficients, as a result of applied distortion correction and/or PET PSF modelling, were tested in paired t-tests. The significance level of all tests was set at 0.05 , and the $95 \%$ CI of the estimated differences were calculated.

\section{Results}

\subsection{FDG-PET: effect of PSF modelling}

The effect of PET PSF modelling is visually depicted in Fig. 1, showing the vendor recommended PET reconstructions with and without PSF modelling. Note, the apparent redistribution of SUV values within the tumor.

The effect of PSF modelling on $\mathrm{SUV}_{\max }$ and $\mathrm{SUV}_{\text {mean }}$ of individual tumors using the vendor recommended image reconstructions is shown in Fig. 2A. As expected, both $S_{U V}$ max and $S_{U V} V_{\text {mean }}$ tend to increase when using PSF modelling: $9 \%$ and $19 \%$ for $S_{U V}$ mean and $S U V_{\text {max }}$, respectively. The relative $S_{U V}$ mean difference versus tumor size, in
Fig. 2B, shows a significantly decreasing trend $(\mathrm{P}=0.01)$.

\subsection{DWI: effect of EPI distortion correction}

DWI sequence optimization and subsequent distortion correction had visible effect on images as seen in the example shown in Fig. 3B-D. Large reductions of distortions were observed for the optimized DWI sequence when compared to the standard DWI. Further improvement of image quality following EPI distortion correction with FUGUE, can also be identified visually.

Fig. 4 illustrates the voxelwise correlation coefficients between the reference STIR image and three types of DWI for each tumor (evaluated in sphere VOI). Based on a paired sample $t$-test, there is a significantly increased correlation when moving from the standard to the optimized DWI (CI of the estimated difference: 0.226 to $0.352, \mathrm{P}<0.001$ ). Similarly, when performing distortion correction with FUGUE on the optimized DWI the correlations to reference are further increased significantly compared to not applying distortion correction (CI: 0.038 to 0.087, $\mathrm{P}<0.001$ ).

\subsection{Spatial correlation of FDG-PET and DWI: Influence of PSF modelling and EPI distortion correction}

Fig. 5 shows the patient with the largest effect of applying PET PSF modelling and EPI distortion correction on the voxelwise correlation coefficient between SUV and ADC. The effect is seen visually on images in the tumor region (Fig. 5B-E) and on the voxelwise correlation (Fig. 5F-G), which changes from non-significant to significant when PET PSF modelling and EPI distortion correction is applied. In the following we investigate, separately, how much each of these two correction methods influences the spatial correlation.

The influence of PET PSF modelling on the spatial relation of FDGPET and DWI can be seen in Fig. 6A, where the paired voxelwise correlation coefficients calculated with and without PET PSF modelling are shown. The differences in correlation coefficients obtained with and without PET PSF modelling were not significantly different from zero (CI: -0.015 to $0.018, \mathrm{P}=0.874$ ). The influence of performing EPI distortion correction by FUGUE on the SUV to ADC correlation coefficients can be seen in Fig. 6B. The correlation coefficients show an increase in magnitude (become more negative) when FUGUE is applied. The difference in the correlation coefficients introduced by EPI distortion correction by FUGUE is statistically significant (CI: -0.085 to $-0.163, \mathrm{P}<0.001)$.

The influence of performing PET PSF modelling and EPI distortion correction on the reproducibility of the spatial relation between SUV and ADC can be seen in Fig. 7. Limits of agreement for the correlation coefficient between SUV and ADC were of the order of \pm 0.3 . Intrapatient variability appears somewhat reduced when applying PSF
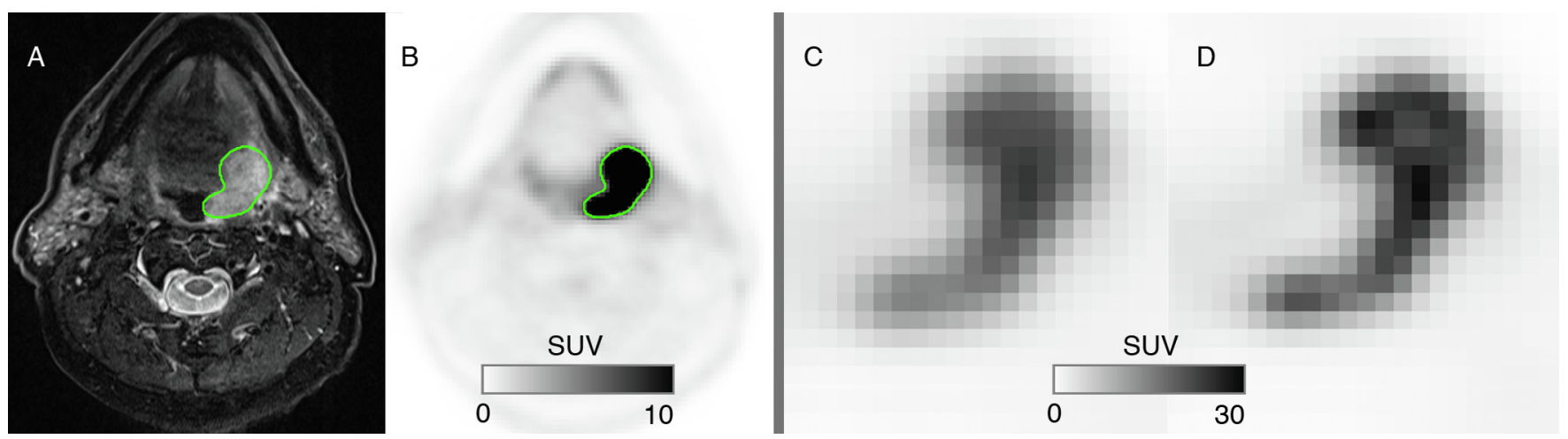

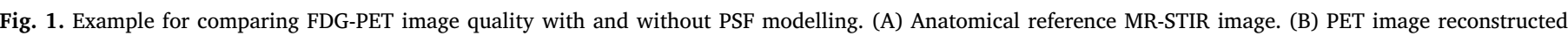

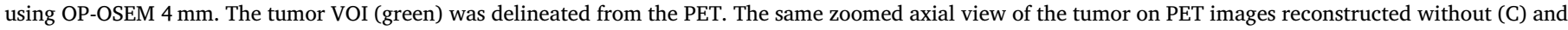

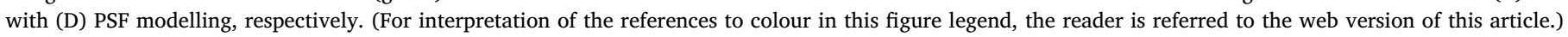


A

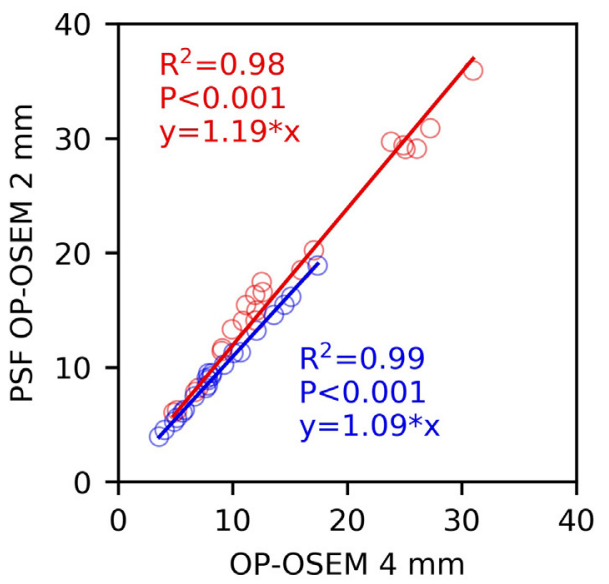

B

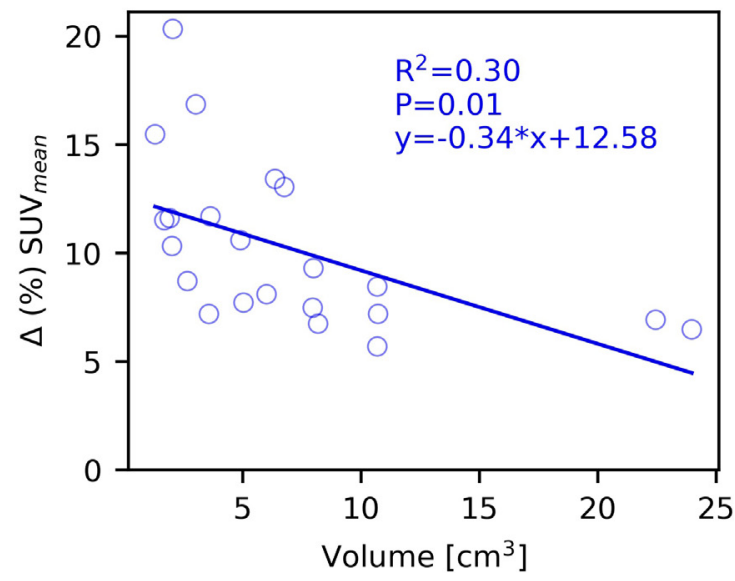

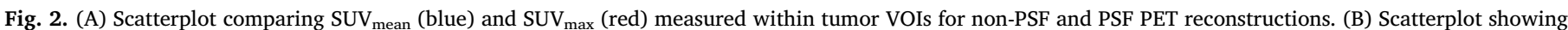

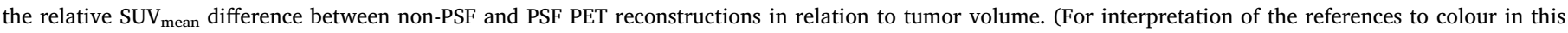
figure legend, the reader is referred to the web version of this article.)

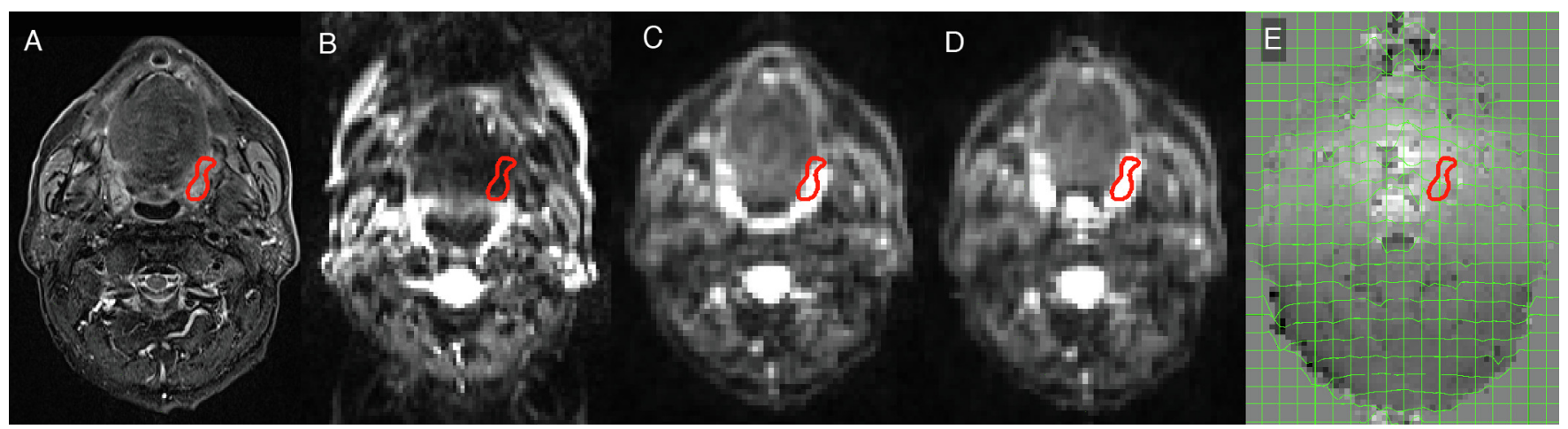

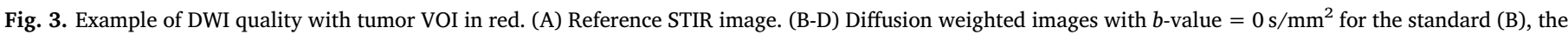

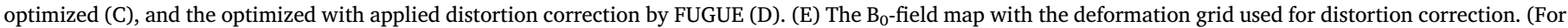
interpretation of the references to colour in this figure legend, the reader is referred to the web version of this article.)

modelling and EPI distortion correction (Fig. 7D) when compared to no processing (Fig. 7A). However, limits of agreement did not differ significantly between methods (confidence intervals for the limits of agreement overlap with estimates for limits of agreement between methods).

\section{Discussion}

This PET/MR imaging study investigated how improving image quality of PET and DWI by PSF modelling and EPI distortion correction, respectively, influences the spatial relation of the modalities. This is highly relevant in view of the potential of combined PET/MR imaging to non-invasively assess tumor heterogeneity [3-7,25,26]. We have demonstrated that the voxelwise correlation between FDG-PET and DWI, quantified as SUV and ADC, was found to be significantly stronger subsequent to EPI distortion correction of the DWI with FUGUE. Notably, this correction was applied to a DWI acquisition protocol, which already was optimized to reduce distortions. In contrast, the correlation did not depend on the PET resolution improvement offered by PSF modelling. Therefore, these findings are important when a voxelwise comparison or modelling of multiparametric imaging data is performed.

Using PSF modelling for PET reconstruction increased SUV $\mathrm{V}_{\text {mean }}$ and SUV $_{\max }$ by $9 \%$ and $19 \%$, respectively. The effect of PSF modelling was, as expected, larger for smaller tumors, as edge effects are more dominant. Only one other study by Aklan, et al. [15] has investigated the quantitative effect of PSF modelling in PET/MR and reported an average increase in $S U V_{\text {mean }}$ and $S U V_{\text {max }}$ of $21 \%$ and $37 \%$, respectively.
However, they reported results from generally smaller lesions located at a larger radial distance from the center of transverse FOV, compared to the present study, which may explain the greater impact of the PET PSF modelling.

The optimization of the DWI protocol resulted in significantly improved imaging compared to the standard DWI. Other oncology studies have reduced distortions by optimizing the EPI-sequence: e.g. by the use of readout-segmented EPI-DWI [19,27]. We note that the echo spacing of the DWI sequence, which is the determining factor for the degree of distortions, is already lower for our optimized sequence than in Bogner et al. and Xia et al. [19,27] (0.145 ms compared to $0.32 \mathrm{~ms}$ and $0.4 \mathrm{~ms}$ ). The current study also demonstrated that performing EPI distortion correction by FUGUE on the optimized DWI protocol further reduced geometric distortions significantly. Distortion correction with $\mathrm{B}_{0}$-field mapping are commonly applied for fMRI [20], but only recently for oncology studies $[8,22,28,29]$. However, with the increasing interest in preserving spatial accuracy, e.g. for radiotherapy purposes, distortion correction of EPI could become even more important [30]. The current study was performed for head and neck cancer where image distortions are known to be severe because of the complex anatomy including bone and air cavities, which enhances magnetic field inhomogeneities [18]. While this serves as a sensitive test of the influence of EPI distortions on multiparametric imaging, other anatomic regions may show less severe artefacts and, in these cases, performing further distortion correction with $\mathrm{B}_{0}$-field mapping may be unnecessary.

The reproducibility of the SUV to ADC correlation coefficient 


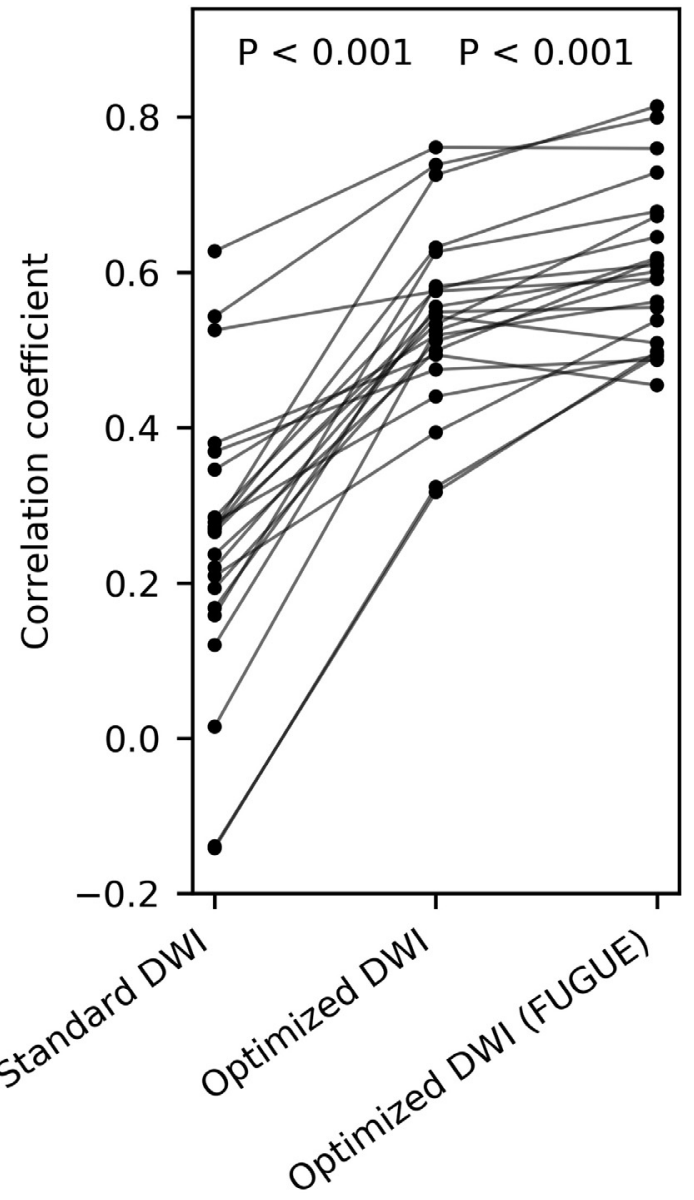

Fig. 4. Paired data for the voxelvise correlation coefficients calculated between the reference STIR image and DWI $\left(b\right.$-value $\left.=0 \mathrm{~s} / \mathrm{mm}^{2}\right)$ of the different sequences.
A

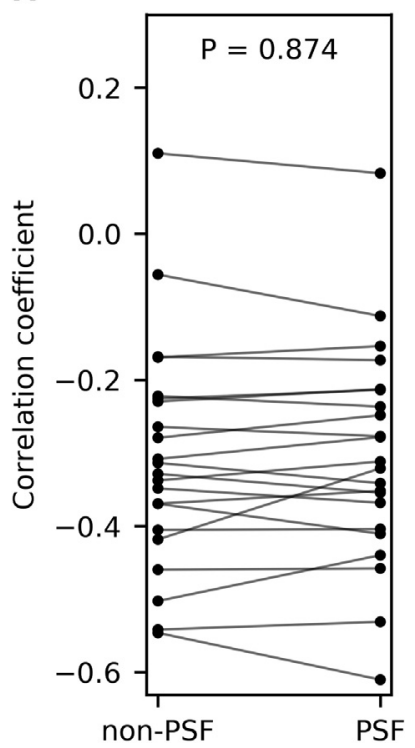

B

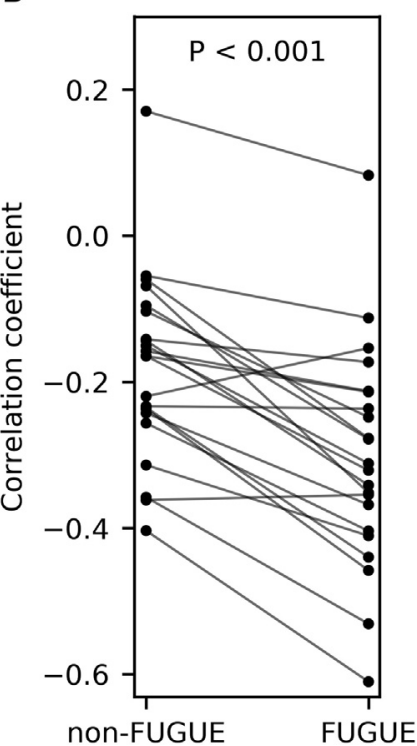

Fig. 6. (A) Paired data for the voxelwise correlations coefficients between ADC derived from optimized DWI corrected for distortion by FUGUE and SUV from PET without and with PSF modelling. (B) Paired data for the voxelwise correlation coefficients between SUV from PET with PSF modelling and ADC derived from optimized DWI without and with distortion correction by FUGUE.

between scan days (Fig. 7) showed a non-significant improving trend when applying PSF modeling and distortion correction. However, in general the correlation coefficient showed a high variability. The low number of patients $(\mathrm{N}=11)$ is clearly a limitation for this analysis. Also, physiological changes occurring between the scan days could affect the reproducibility. We note that the multiparametric analyses carried out here used a Spearman's correlation coefficient. Consequences for a more advanced analysis, e.g. clustering analysis as carried out in [5-8], was not pursued. Such analyses may provide a
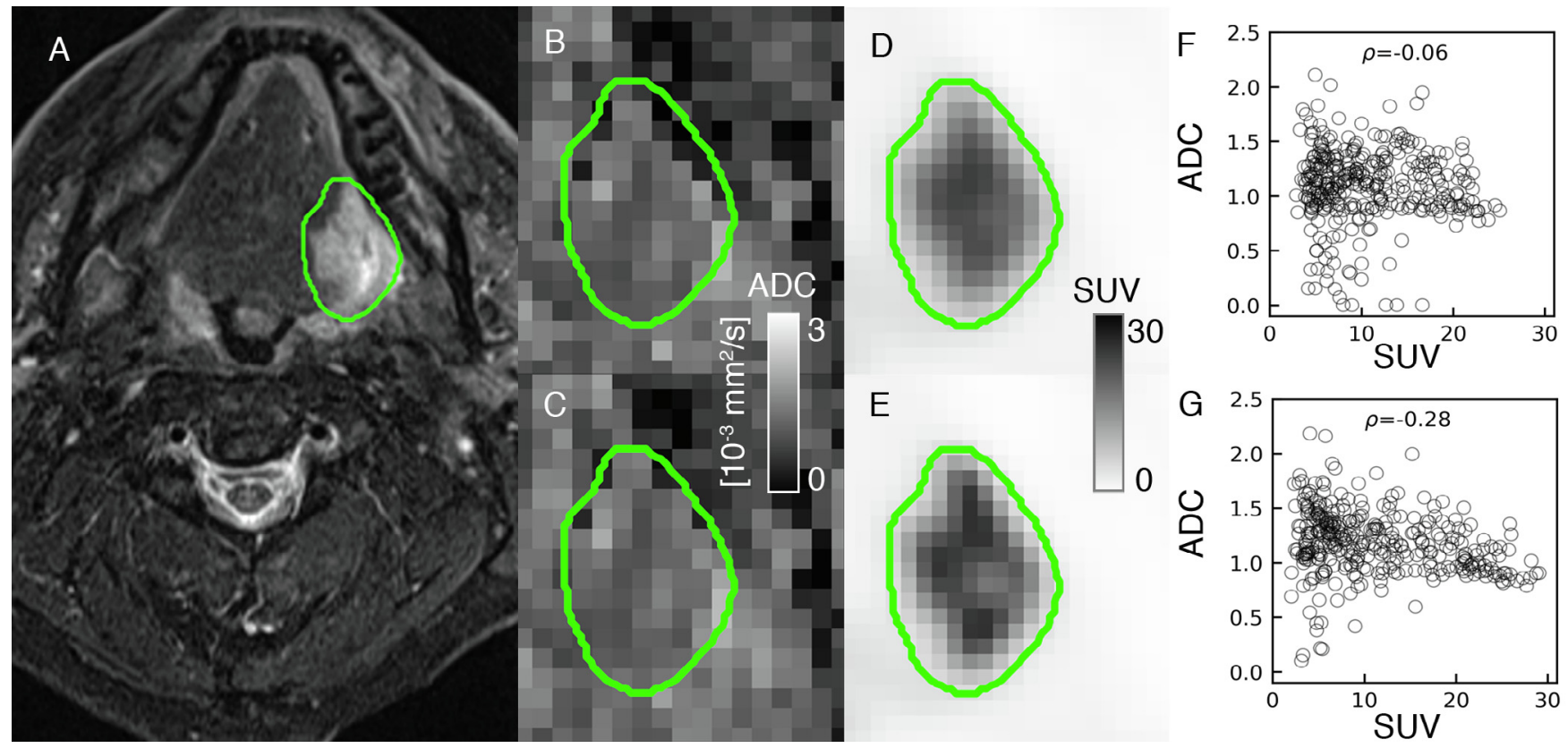

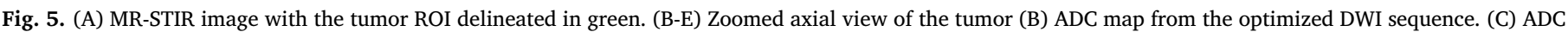

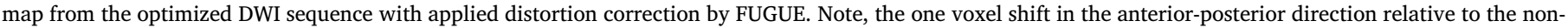

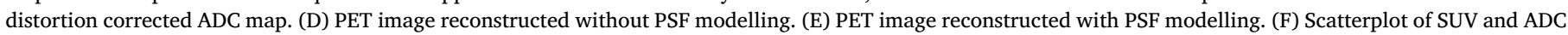

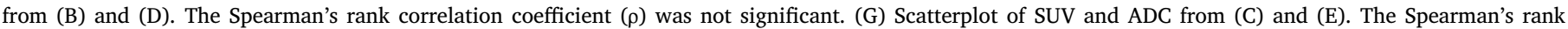

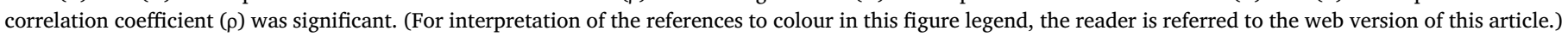




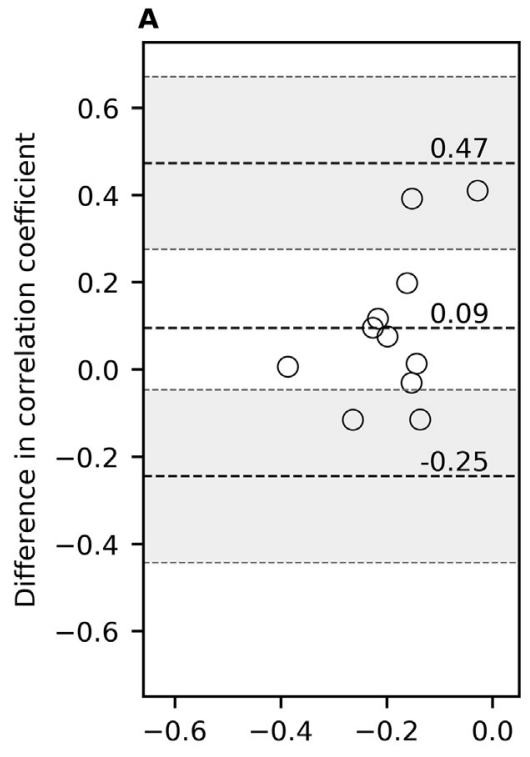

B

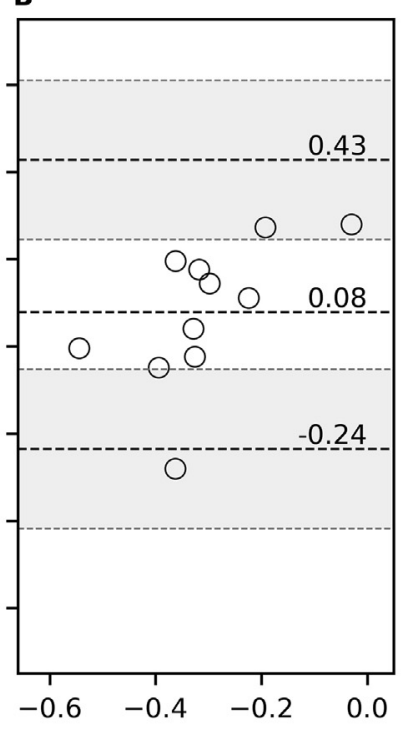

C

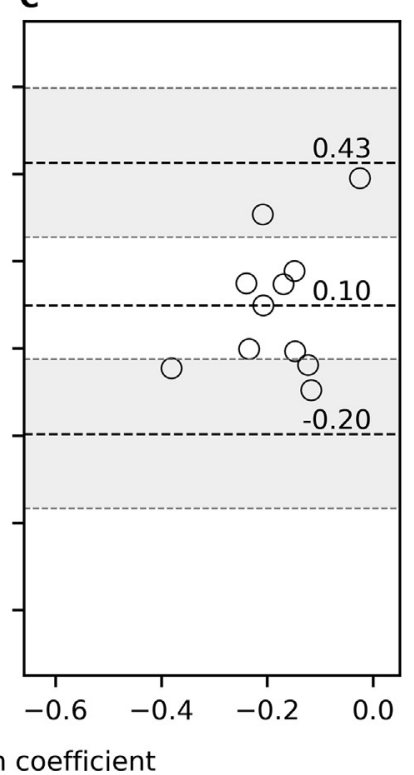

D

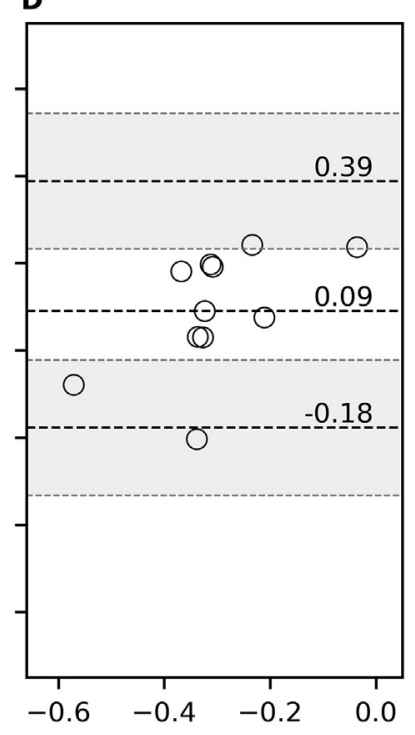

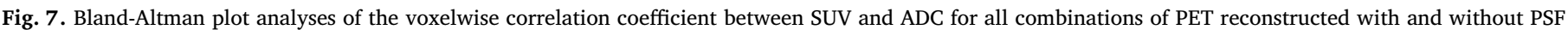

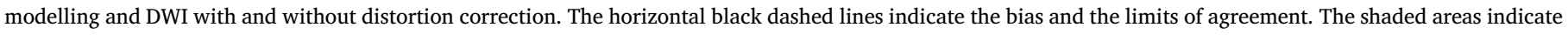

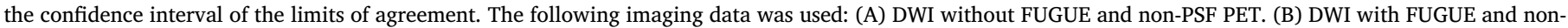
PSF PET. (C) DWI without FUGUE and PSF PET. (D) DWI with FUGUE and PSF PET.

more stable metric for multiparametric tumor characterization than the simple metric studied here. Larger, systematic studies are needed to firmly characterize the reproducibility of multiparametric measures.

Further limitations of this study include the lack of spatial histology verification as in Schmitz, et al. [7]. Therefore, a histologic verification of the possible importance of a correlation between ADC to SUV was not possible. Concerning correction of DWI distortions, a morphological gold standard was not available. We instead computed the spatial correlation of the DWI $b$-value $=0 \mathrm{~s} / \mathrm{mm}^{2}$ to a T2-weighted image, which is much more robust to distortions than DWI based on EPI, while providing similar image contrast. A similar approach to quantify distortions was used by Vardal et al. [29]. The method chosen for VOI definition is frequently applied in the literature; however, it may result in a bias as tumors are defined by data from non-PSF PET only. The head and neck cancer lesions in this study showed qualitatively only little heterogeneity. We still observed an effect of distortion correction on the SUV to ADC correlation coefficients, but the effect might be larger in more heterogeneous tumors. For the analysis of the effect of PSF modelling on the SUV to ADC correlation coefficients, PET data was resampled to match the ADC voxels, which could slightly reduce the PSF resolution enhancement. Finally, for all t-tests, data from both scan days for each patient were included, violation the assumption of independent data. To verify our results, all tests were reperformed using only data from one of the scan days (results not shown), and all statistical conclusions remained unchanged.

\section{Conclusion}

This study investigated the consequences of improving quality of PET and DWI for the spatial correlation of the modalities evaluated for head and neck cancer patients undergoing combined FDG-PET/MR imaging. The magnitude of the voxelwise correlation between tumor SUV from FDG PET and ADC from DWI increased significantly after distortion correction of the DWI. However, the correlation was not found to be influenced by PSF modelling in the reconstruction of PET. In the setting of PET/MR of head and neck cancer, proper preparation of the imaging modalities is important for a correct interpretation of a voxelwise multiparametric analysis.

\section{Acknowledgement}

We kindly thank the John and Birthe Meyer Foundation for donating the PET/MR system to Rigshospitalet.

\section{Disclosure}

All authors declare that they have no conflict of interest.

\section{References}

[1] Tannock IF, Hickman JA. Limits to personalized cancer medicine. N Engl J Med 2016;375:1289-94.

[2] Koh D-MM, Collins DJ. Diffusion-weighted MRI in the body: applications and challenges in oncology. Am J Roentgenol 2007;188:1622-35. https://doi.org/10. 2214/AJR.06.1403.

[3] Houweling AC, Wolf AL, Vogel WV, Hamming-Vrieze O, van Vliet-Vroegindeweij C, van de Kamer JB, et al. FDG-PET and diffusion-weighted MRI in head-and-neck cancer patients: implications for dose painting. Radiother Oncol 2013:106:250-4.

[4] Leibfarth S, Simoncic U, Mönnich D, Welz S, Schmidt H, Schwenzer N, et al. Analysis of pairwise correlations in multi-parametric PET/MR data for biological tumor characterization and treatment individualization strategies. Eur J Nucl Med Mol Imaging 2016;43:1199-208.

[5] Schmidt H, Brendle C, Schraml C, Martirosian P, Bezrukov I, Hetzel J, et al. Correlation of simultaneously acquired diffusion-weighted imaging and 2-deoxy[18F] fluoro-2-D-glucose positron emission tomography of pulmonary lesions in a dedicated whole-body magnetic resonance/positron emission tomography system. Invest Radiol 2013;48:247-55.

[6] Divine MR, Katiyar P, Kohlhofer U, Quintanilla-Martinez L, Pichler BJ, Disselhorst JA. A population-based Gaussian mixture model incorporating 18F-FDG PET and diffusion-weighted MRI quantifies tumor tissue classes. J Nucl Med 2016;57:473-9. https://doi.org/10.2967/jnumed.115.163972.

[7] Schmitz J, Schwab J, Schwenck J, Chen Q, Quintanilla-Martinez L, Hahn M, et al Decoding intratumoral heterogeneity of breast cancer by multiparametric in vivo imaging: a translational study. Cancer Res 2016.

[8] Rasmussen JH, Nørgaard M, Hansen AE, Vogelius IR, Aznar MC, Johannesen HH, et al. Feasibility of multiparametric imaging with PET/MR in head and neck squamous cell carcinoma. J Nucl Med 2017;58:69-74. https://doi.org/10.2967/ jnumed.116.180091.

[9] Gillies RJ, Beyer T. PET and MRI: is the whole greater than the sum of its parts? Cancer Res 2016;76:6163-6.

[10] Watson CC. Measurement of the physical PSF for an integrated PET/MR using targeted positron beams. Nucl. Sci. Symp. Med. Imaging Conf. (NSS/MIC), 2012 IEEE. IEEE; 2012. p. 2089-95.

[11] Panin VY, Kehren F, Michel C, Casey M. Fully 3-D PET reconstruction with system matrix derived from point source measurements. IEEE Trans Med Imaging 2006;25:907-21. 
[12] Alessio AM, Kinahan PE, Lewellen TK. Modeling and incorporation of system response functions in 3-D whole body PET. IEEE Trans Med Imaging 2006;25:828-37.

[13] Andersen FL, Klausen TL, Loft A, Beyer T, Holm S. Clinical evaluation of PET image reconstruction using a spatial resolution model. Eur J Radiol 2013;82:862-9.

[14] Petibon Y, Huang C, Ouyang J, Reese TG, Li Q. Relative role of motion and PSF compensation in whole-body oncologic PET-MR imaging. Med Phys 2014;41:1-12. https://doi.org/10.1118/1.4868458.

[15] Aklan B, Oehmigen M, Beiderwellen K, Ruhlmann M, Paulus DH, Jakoby BW, et al. Impact of point-spread function modeling on PET image quality in integrated PET/ MR hybrid imaging. J Nucl Med 2016;57:78-84. https://doi.org/10.2967/jnumed. 115.154757.

[16] Jezzard P. Correction of geometric distortion in fMRI data. Neuroimage 2012;62:648-51. https://doi.org/10.1016/j.neuroimage.2011.09.010.

[17] Schenck JF. The role of magnetic susceptibility in magnetic resonance imaging: MRI magnetic compatibility of the first and second kinds. Med Phys 1996;23:815-50.

[18] Thoeny HC, De Keyzer F, King AD. Diffusion-weighted MR imaging in the head and neck. Radiology 2012;263:19-32.

[19] Bogner W, Pinker-Domenig K, Bickel H, Chmelik M, Weber M, Helbich TH, et al. Echo-pIanar imaging improves the diagnostic performance of diffusion-weighted MR breast examinations at 3.0 T. Radiology 2012;263:64-76. https://doi.org/10. 1148/radiol.12111494/-/DC1.

[20] Jezzard P, Balaban RS. Correction for geometric distortion in echo planar images from B0 field variations. Magn Reson Med 1995;34:65-73.

[21] Hutton C, Bork A, Josephs O, Deichmann R, Ashburner J, Turner R. Image distortion correction in fMRI: a quantitative evaluation. Neuroimage 2002;16:217-40.

[22] Hansen AE, Rasmussen JH, Johannesen HH. Geometric distortions of diffusion weighted imaging of the head/neck in combined PET/MR: optimization of image acquisition and post-processing correction for oncology applications [Brief communication]. EJNMMI Phys 2014. p. 1:A76.

[23] Rasmussen JH, Fischer BM, Aznar MC, Hansen AE, Vogelius IR, Löfgren J, et al. Reproducibility of 18F-FDG PET uptake measurements in head and neck squamous cell carcinoma on both PET/CT and PET/MR. Br J Radiol 2015;88:20140655. https://doi.org/10.1259/bjr.20140655.

[24] Bland JM, Altman DG. Statistical methods for assessing agreement between two methods of clinical measurement. Int J Nurs Stud 2010;47:931-6. https://doi.org/ 10.1016/j.ijnurstu.2009.10.001.

[25] Eiber M, Nekolla SG, Maurer T, Weirich G, Wester H-J, Schwaiger M. 68 Ga-PSMA PET/MR with multimodality image analysis for primary prostate cancer. Abdom Imaging 2015;40:1769-71.

[26] Metz S, Ganter C, Lorenzen S, van Marwick S, Holzapfel K, Herrmann K, et al. Multiparametric MR and PET imaging of intratumoral biological heterogeneity in patients with metastatic lung cancer using voxel-by-voxel analysis. PLoS One 2015; 10:e0132386.

[27] Xia C, Liu X, Peng W, Li L, Zhang J, Meng W, et al. Readout-segmented echo-plana imaging improves the image quality of diffusion-weighted MR imaging in rectal cancer: comparison with single-shot echo-planar diffusion-weighted sequences. Eur J Radiol 2016;85:1818-23.

[28] Winter RM, Schmidt H, Leibfarth S, Zwirner K, Welz S, Schwenzer NF, et al. Distortion correction of diffusion-weighted magnetic resonance imaging of the head and neck in radiotherapy position. Acta Oncol (Madr) 2017;56:1659-63.

[29] Vardal J, Salo RA, Larsson C, Dale AM, Holland D, Groote IR, et al. Correction of BOdistortions in echo-planar-imaging - based perfusion-weighted MRI. J Magn Reson Imaging 2014;39:722-8. https://doi.org/10.1002/jmri.24213.

[30] Thorwarth D, Leibfarth S, Mönnich D. Potential role of PET/MRI in radiotherapy treatment planning. Clin Transl Imaging 2013;1:45-51. https://doi.org/10.1007/ s40336-013-0006-2. 